\section{A BRUSH FOR PATIENTS IN A RUSH}

The Cavex Rush Brush is a ready to use toothbrush impregnated with toothpaste. It is aimed at the patient who has not had time to clean their teeth before visiting the surgery or as an inexpensive way to demonstrate cleaning and brushing technique.

Each Rush Brush is individually packaged for convenience and the small head size allows excellent access to all areas of the mouth. Although inexpensive the Rush Brush has a

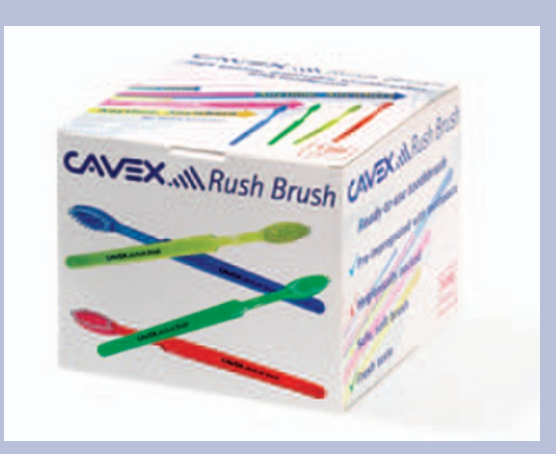

fresh mint taste and soft quality bristles which are kind to gums. A box of 100 brushes costs $£ 15$ + VAT (RRP). Reader response number 59

\section{STOP, DRAW AND TEACH}

guru, an interactive patient education tool from Software of Excellence, uses a unique 'stop, draw and teach technology' to help dentists and hygienists explain why a particular treatment is their preferred recommendation, effectively increasing treatment plan acceptance.

This standalone computer based technology enables practitioners to customise patient presentations by incorporating individual patient X-rays, intraoral and extraoral images. The patient explanation can also be augmented by pausing the animation and annotating directly onto the screen via a tablet and stylus, or by recording an audio explanation that addresses the specific needs of the individual case. Presentations can then

\section{PATIENT SELF-DIAGNOSIS TOOL}

MyDentalScore has been devised to educate patients about their dental health. It consists of an online questionnaire which asks about dental hygiene and history as well as lifestyle and delivers a score along with encouragement to discuss the findings with a dental professional. It covers the three main aspects of oral health: teeth, gums and oral cancer.

MyDentalScore can be branded to a practice and a link to the practice website can be added.

MyDentalScore is distributed by Oral Health Innovations (OHI) Ltd who also be printed, emailed or burnt to a DVD or memory stick for the patient to take away.

The guru library contains more than 200 excellent quality 3D animations on a range of treatment options including oral health, implants, periodontal disease and orthodontics and is constantly being updated and refined.

\section{Reader response number 60}

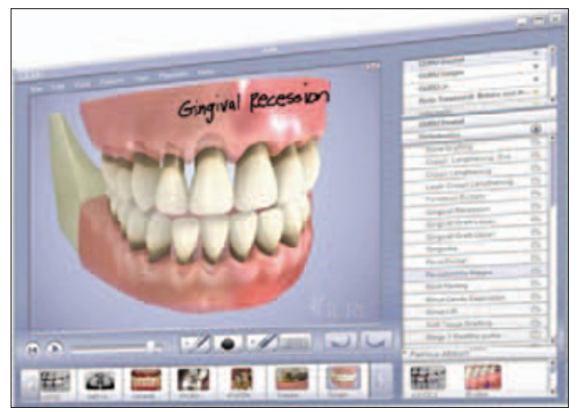

devised PreViser, a periodontal risk assessment tool which uses clinical measurements and is managed by the dental professional. OHI's profits are divided between the UK Oral and Dental Research Trust and grants to PhD students at Birmingham's Dental School.

Two formats of MyDentalScore are available: one to be hosted on dental practice websites and a second, public version which will list by postcode PreViser-capable dentists who can clinically validate the patients' self assessed results and contextualise the results with their own advice and guidance.

Reader response number 61 